\title{
Tradução e adaptação cultural do questionário sobre motivo das escolhas alimentares (Food Choice Questionnaire - FCQ) para a língua portuguesa
}

\author{
Translation and cultural adaptation of the questionnaire \\ on the reason for food choices (Food Choice Questionnaire - FCQ) \\ into Portuguese
}

\author{
Sara Franco Diniz Heitor ${ }^{1}$ \\ Camilla Chermont Prochnik Estima ${ }^{2}$ \\ Fabricia Junqueira das Neves ${ }^{3}$ \\ Aline Silva de Aguiar ${ }^{4}$ \\ Sybelle de Souza Castro ${ }^{5}$ \\ Julia Elba de Souza Ferreira ${ }^{1}$
}

${ }^{1}$ Departamento de Nutrição, Centro

Educacional, Universidade Federal do Triângulo Mineiro (UFTM). Avenida Getúlio Guaritá 159/321, Abadia. 38025-440 Uberaba MG.Brasil. jesferreira@ globo.com

${ }^{2}$ Fundação Carlos Chagas Filho de Amparo à Pesquisa do Estado do RJ.

${ }^{3}$ Departamento de

Nutrição Aplicada, Escola de Nutrição, Universidade

Federal do Estado do Rio de Janeiro.

${ }^{4}$ Departamento de Nutrição, Universidade Federal de Juiz de Fora. ${ }^{5}$ Departamento de Medicina Social, UFTM.

\begin{abstract}
The Food Choice Questionnaire (FCQ) assesses the importance that subjects attribute to nine factors related to food choices: health, mood, convenience, sensory appeal, natural content, price, weight control, familiarity and ethical concern. This study sought to assess the applicability of the FCQ in Brazil; it describes the translation and cultural adaptation from English into Portuguese of the FCQ via the following steps: independent translations, consensus, back-translation, evaluation by a committee of experts, semantic validation and pre-test. The pre-test was run with a randomly sampled group of 86 male and female college students from different courses with a median age of 19. Slight differences between the versions were observed and adjustments were made. After minor changes in the translation process, the committee of experts considered that the Brazilian Portuguese version was semantically and conceptually equivalent to the English original. Semantic validation showed that the questionnaire is easily understood. The instrument presented a high degree of internal consistency. The study is the first stage in the process of validating an instrument, which consists of face and content validity. Further stages, already underway, are needed before other researchers can use it.
\end{abstract}

Key words Translating, Cross-Cultural comparison, Food preferences, Validation Studies
Resumo O Food Choice Questionnaire (FCQ) avalia a importância atribuída pelos indivíduos a nove fatores relacionados às escolhas alimentares: saúde, humor, conveniência, apelo sensorial, conteúdo natural, preço, controle de peso, familiaridade e preocupação ética. O estudo objetivou descrever o processo de tradução e adaptação cultural do FCQ para a língua portuguesa e avaliar sua aplicabilidade com as seguintes etapas: traduções independentes, obtenção do consenso em português, retrotradução, avaliação por um comitê de especialistas, validação semântica e pré-teste. Este foi aplicado em uma amostra aleatória de 86 estudantes universitários, de ambos os sexos, mediana de 19 anos de idade. Pequenas diferenças entre as versões foram observadas e poucas adaptações realizadas. Após discretas modificações nos processos de tradução, o comitê de especialistas considerou que a versão para o português apresentou equivalências semântica e conceitual. A validação semântica demonstrou fácil compreensão. $O$ instrumento apresentou alto grau de consistência interna. O estudo foi a primeira etapa do processo de validação de um instrumento, que é a validade de face e de conteúdo. Novas etapas, que já se encontram em andamento, são necessárias antes de sua utilização por outros pesquisadores.

Palavras-chave Tradução, Comparação transcultural, Preferências alimentares, Estudos de validação 


\section{Introdução}

Os motivos que norteiam as escolhas alimentares das pessoas baseiam-se em vários aspectos. Primeiramente, poder-se-ia pensar na condição onívora do ser humano. Todavia, somente isto não determina o comportamento alimentar de um indivíduo, tendo em vista que há uma série de fatores, tais como os socioculturais, econômicos, psicológicos, além dos biológicos, que irão determinar as preferências alimentares ${ }^{1}$.

A preocupação com as escolhas alimentares e a sua possível relação com efeitos adversos sobre a saúde está incorporada tanto em documentos nacionais ${ }^{2-4}$, como nos internacionais ${ }^{5}$ e direcionam nossas políticas públicas na área de alimentação e nutrição. Recomendações para restringir o consumo de sal, gordura e produtos açucarados e ter uma ingestão regular de frutas, legumes e verduras, a fim de diminuir o risco de ocorrência de doenças crônicas não transmissíveis, são princípios centrais desses documentos ${ }^{2-5}$. Entretanto, o consumo dos alimentos ultra processados, de alta densidade energética, permanece elevado no Brasil $^{6-8}$ e esse padrão alimentar se correlaciona com as taxas de excesso de peso, obesidade e distúrbios metabólicos ${ }^{6,7}$. Tal paradoxo reforça a questão de que orientações na área da alimentação e nutrição pautadas somente pelo prisma da saúde parecem ser limitadas ${ }^{9}$.

Ressalta-se que já existem iniciativas de aproximação e diálogo entre outras áreas, que não seja apenas a nutrição, como por exemplo, com as ciências sociais, demonstrando que estudos acerca da alimentação necessitam de uma abordagem multidimensional, que articulem além das dimensões biológicas, as socioeconômicas, culturais e ideológicas que as pessoas vivenciam no seu cotidiano ${ }^{10}$.

Assim, a busca pela compreensão de alguns importantes aspectos que possam nortear as escolhas alimentares é relevante para que ações de educação alimentar e nutricional possam ser desenvolvidas com maior efetividade ${ }^{3}$, ainda que esses aspectos sejam complexos, interajam entre si e se modifiquem com o tempo.

Para tanto, é importante discutir sobre a necessidade de abordagens metodológicas pluridisciplinares e de instrumentos que sejam úteis para melhorar a compreensão dos motivos que levam as escolhas alimentares ${ }^{9,11}$.

Pesquisas pautadas em abordagens metodológicas qualitativas costumam investigar com primor os determinantes das escolhas alimentares de indivíduos. No entanto, tais estudos abrangem um número restrito de pessoas e, pelas características de suas amostras, que não se baseiam em cálculos numéricos, mas sim na saturação de suas respostas ${ }^{12}$, não podem ter seus resultados extrapolados como comumente é feito em pesquisas com amostras probabilísticas. Sendo assim, estudos epidemiológicos voltados para investigação dos determinantes das escolhas alimentares necessitariam de instrumentos sistematizados, como por exemplo, questionários autoaplicáveis, com respostas fechadas.

No que tange a instrumentos validados para uso no Brasil, que visam compreender o amplo espectro que norteia as escolhas alimentares das pessoas, esses não foram encontrados após ampla revisão da literatura realizada pelos autores, apesar da existência de inúmeros instrumentos que se propõem a quantificar ou captar o consumo usual dos indivíduos, como por exemplo, os questionários de frequência de consumo alimentar. Contudo, esses questionários, vastamente utilizados em estudos epidemiológicos, não se propõem a compreender os motivos das escolhas alimentares.

Todavia, Steptoe et al. ${ }^{9}$, em Londres, desenvolveram o Food Choice Questionnaire para população adulta, um questionário cujo objetivo é avaliar a importância atribuída pelos indivíduos a uma série de aspectos relacionados às escolhas alimentares, tais como: saúde, humor, conveniência, aspectos sensoriais do alimento, preço, familiaridade, preocupação com o peso corporal, entre outros. Este questionário já foi utilizado em vários estudos na Europa ${ }^{13-16}$ e somente em uma pesquisa Latino Americana, realizada no Uruguai ${ }^{17}$. No entanto, ainda não foi traduzido e adaptado à língua portuguesa no Brasil.

Uma vez que o questionário supracitado poderá ser útil na investigação dos determinantes das escolhas alimentares em estudos epidemiológicos brasileiros, torna-se pertinente a tradução e a adaptação cultural do mesmo para a língua portuguesa. Além disso, sua utilização permitirá a comparação entre pesquisas realizadas no Brasil e os achados internacionais ${ }^{13-17}$.

Sendo assim, o objetivo do presente estudo foi descrever o processo de adaptação cultural do Food Choice Questionnaire (Questionário sobre Motivo das Escolhas Alimentares) para a língua portuguesa e sua aplicabilidade. 


\section{Métodos}

O FCQ ${ }^{9}$ é um instrumento autoaplicável, desenvolvido originalmente na língua inglesa, composto por 36 itens que são distribuídos em nove fatores, ou dimensões, que avaliam aspectos relacionados às escolhas alimentares, conforme explicitado no Quadro 1.

O participante é convidado a refletir sobre a declaração o Para mim é importante que o alimento que eu coma no dia-a-dia seja..., para posteriormente indicar, dentro de cada fator os itens que refletem o que ele considera ser mais importante para determinar sua escolha alimentar. As opções de resposta são apresentadas em uma escala do tipo likert $^{18}$ e variam de 1 a 4 pontos: 1 (nada importante); 2 (um pouco importante); 3 (moderadamente importante) e 4 (muito importante), gerando desta forma um escore. Assim, calcula-se uma média não ponderada para cada fator do questionário. Com isso, pontuações mais elevadas indicam que o participante atribuiu maior importância a determinado fator.

Para iniciar o processo de tradução, primeiramente, obteve-se a permissão para utilização do FCQ, por meio de correio eletrônico junto ao primeiro autor do instrumento. Em seguida, utilizaram-se as etapas metodológicas propostas por Guillemin et al. ${ }^{19}$ e Beaton et al..$^{20,21}$, para realização da adaptação cultural do FCQ para a língua portuguesa, conforme descrito a seguir.

\section{1 a Etapa: Tradução e retrotradução}

Duas nutricionistas professoras doutoras com fluência no idioma inglês e experiência na temática abordada e uma terceira tradutora bilíngue sem experiência na área da saúde e sem manterem contato entre si realizaram traduções independentes. As três versões em português foram comparadas e discutidas por duas pesquisadoras nutricionistas envolvidas no projeto, uniformizando o uso de expressões divergentes, elaborando-se assim, a primeira versão consensual do instrumento em português (VP1).

$\mathrm{Na}$ sequência, dois tradutores nativos na língua inglesa, residentes há anos no Brasil, que não tinham conhecimento dos objetivos do estudo e nem da versão original do instrumento, fizeram a tradução reversa da VP1. Cada um deles elaborou uma versão independente em inglês. As duas pesquisadoras nutricionistas envolvidas no projeto discutiram com os tradutores nativos, apresentaram os objetivos do estudo e sua aplicabilidade, e após ajustes, houve concordância entre as duas versões, definindo a versão final em inglês (VIF). Neste momento a VIF foi comparada com a VP1, ajustes foram feitos na versão em português, obtendo-se assim o segundo consenso da versão em português do instrumento (VP2). Após estas etapas, a VIF foi encaminhada ao primeiro autor do instrumento ${ }^{9}$, para aprovação ou sugestões caso necessário. Este respondeu que as pequenas diferenças de redação não causariam problemas, aceitando a retrotradução do instrumento em sua totalidade.

\section{2a Etapa: Avaliação por um comitê de especialistas}

Um grupo composto por sete pessoas foi formado, sendo um especialista em instrumentos de medida, uma professora doutora do curso de Nutrição de uma Universidade Federal, uma nutricionista com experiência na área, uma psicóloga professora doutora do curso de Psicologia de uma Universidade Federal, uma pessoa convidada representando um potencial indivíduo que será investigado e duas pesquisadoras com experiência em docência, todos com domínio da língua inglesa. $\mathrm{O}$ grupo foi informado que o objetivo desse comitê era avaliar as equivalências semântica, idiomática, cultural e conceitual dos itens da versão traduzida - VP2, assim como analisar a pergunta norteadora do instrumento e rever a escala de respostas, mantendo a equivalência de medidas. Dessa maneira, cada membro do comitê recebeu a versão original do questionário, juntamente com a VP2, e responderam individualmente um formulário de análise proposto pelos pesquisadores do grupo DISABKIDS ${ }^{22}$, que comparava cada item com o do questionário original, discutindo-se, então, cada um dos 36 itens, verificando-se desta forma a pertinência ou não da tradução. As questões avaliadas pelos especialistas $^{22}$ foram: Isso é relevante para você? Você tem dificuldade para entender essa questão? As opções de resposta estão claras e de fácil entendimento? Como você falaria/expressaria isso, caso tenha dúvidas?

Alterações na redação foram feitas quando não houve concordância com a tradução. Já a aprovação ocorreu quando cinco participantes (mais que $80 \%$ ) ou mais concordavam com a mudança. Com a conclusão dessa etapa, obtevese o terceiro consenso da versão em português do instrumento (VP3). 
Quadro 1. Síntese da versão traduzida e avaliação das equivalências semântica, idiomática, cultural e conceitual pelo comitê de especialistas para os itens do Food Choice Questionnaire (FCQ).

\begin{tabular}{|c|c|c|}
\hline $\begin{array}{l}\text { Documento } \\
\text { Original }\end{array}$ & $\begin{array}{c}\text { Síntese } \\
\text { da versão traduzida }\end{array}$ & $\begin{array}{l}\text { Versão final após avaliação } \\
\text { por comitê de especialistas }\end{array}$ \\
\hline $\begin{array}{l}\text { Food Choice Questionnaire } \\
\text { It is important to me that the } \\
\text { food I eat on a typical day: }\end{array}$ & $\begin{array}{l}\text { Motivo para escolhas alimentares } \\
\text { Para mim é importante que o } \\
\text { alimento que eu coma no dia-a-dia: }\end{array}$ & $\begin{array}{l}\text { Motivo para escolhas alimentares } \\
\text { Para mim é importante que o } \\
\text { alimento que eu coma no dia-a-dia: }\end{array}$ \\
\hline Factor 1 - Health & Fator 1 - Saúde & Fator 1 - Saúde \\
\hline 1. Contains a lot of vitamins and & grande quantidade & Contenha uma grande quantidade \\
\hline minerals & de & de vi \\
\hline 2. Keeps me healthy & Me man & Me m \\
\hline ritious & & $\mathrm{S}$ \\
\hline 4. Is high in protein & uita proteína & Tenha muita proteína \\
\hline $\begin{array}{l}\text { 5. Is good for my skin/teeth/hair/ } \\
\text { nails etc }\end{array}$ & $\begin{array}{l}\text { Seja bom para a minha pele/dentes/ } \\
\text { cabelos / unhas, etc. }\end{array}$ & $\begin{array}{l}\text { Seja bom para a minha pele/dentes/ } \\
\text { cabelos / unhas, etc. }\end{array}$ \\
\hline 6. Is high in fibre and roughage & Seja rico em fibra e me dê saciedade. & Seja rico em fibra e me dê saciedade \\
\hline Factor 2 - Mood & Fator 2 & Fator 2 \\
\hline ith stress & idar com o estresse & Me ajude a lidar com o estresse \\
\hline 8. Helps me to col & de a lidar c & Me ajude a lidar com \\
\hline lns me relay & de relayr & Me ajude a relaxar \\
\hline 10. $\mathrm{I}$ & $\mathrm{Me}$ & (a) / alerta \\
\hline 11. & M & M \\
\hline $12 . \mathrm{N}$ & bem & bem \\
\hline Factor 3 & Fat & Fator 3 - Conveniência \\
\hline 13. & Se & Seja \\
\hline 14. $\mathrm{Ca}$ & $\begin{array}{l}\text { Possa ser cozinhado de forma muito } \\
\text { simples }\end{array}$ & $\begin{array}{l}\text { Possa ser cozinhado de forma muito } \\
\text { simples }\end{array}$ \\
\hline 15. Takes no time to $\mathrm{F}$ & $\begin{array}{l}\text { Não leve muito tempo para ser } \\
\text { preparado }\end{array}$ & $\begin{array}{l}\text { Não leve muito tempo para ser } \\
\text { preparado }\end{array}$ \\
\hline 16. & & ocais perto \\
\hline to $\mathrm{r}$ & & \\
\hline $\begin{array}{l}\text { 17. Is easily available in shops and } \\
\text { supermarkets }\end{array}$ & $\begin{array}{l}\text { Seja fácil de achar em mercearias e } \\
\text { supermercados }\end{array}$ & $\begin{array}{l}\text { Seja fácil de achar em mercearias e } \\
\text { supermercados }\end{array}$ \\
\hline ensory Appeal & elo Sensorial & Fator 4 - Apelo Sensorial \\
\hline Nice & Ten & $\mathrm{Te}$ \\
\hline Tice & $\mathrm{Te}$ & ncia \\
\hline exture & ável & a textura \\
\hline 21. & $\mathrm{Se}$ & \\
\hline Factor 5 - Natur & nteúdo Natural & Fator 5 - Conteúdo Natural \\
\hline ditives & tóxicos ${ }^{*}$ & Nã \\
\hline ingredients & $\mathrm{Co}$ & Contenha ingredie \\
\hline $\begin{array}{l}\text { 24. Contains no artificial } \\
\text { ingredients }\end{array}$ & Não contenha ingredientes artificiais & $\begin{array}{l}\text { ntenha ingredientes } \\
\text { is }\end{array}$ \\
\hline Facto & Fato & 6 - Preço \\
\hline expensive & $\mathrm{N}$ & $\mathrm{N}$ \\
\hline 26 & $\mathrm{Se}$ & arato \\
\hline oney & $\mathrm{Ter}$ & Tenha o preço ju \\
\hline Factor 7 - Weight Control & Fato & Fator 7 -Controle de peso \\
\hline 28. Is low in calories & Tenha poucas calorias & Tenha poucas calorias \\
\hline ntrol my weight & neu peso & Me ajude a controlar o meu peso \\
\hline & $\mathrm{Te}$ & Tenha pouca gordura \\
\hline iliarity & Familiarid & Fator 8 - Familiaridade \\
\hline s what I usually eat & e eu costumo comer & Seja o que eu costumo co \\
\hline & & Sej \\
\hline $\begin{array}{l}\text { 33. Is like the food I ate when I } \\
\text { was a child }\end{array}$ & $\begin{array}{l}\text { Seja parecido com a comida que eu } \\
\text { comia quando era crianca }\end{array}$ & $\begin{array}{l}\text { Seja parecido com a comida que eu } \\
\text { comia quando era crianca }\end{array}$ \\
\hline or 9 - Ethical Concern & Fator 9 - Preocupação ética & Fator 9 - Preocupação ética \\
\hline $\begin{array}{l}\text { 34. Comes from countries I } \\
\text { approve of politically }\end{array}$ & $\begin{array}{l}\text { Venha de países que eu aprove } \\
\text { a forma como os alimentos são } \\
\text { produzidos }\end{array}$ & $\begin{array}{l}\text { Venha de países que eu aprove } \\
\text { a forma como os alimentos são } \\
\text { produzidos }\end{array}$ \\
\hline $\begin{array}{l}\text { 35. Has the country of origin } \\
\text { clearly marked }\end{array}$ & $\begin{array}{l}\text { Mostre com clareza, a identificação } \\
\text { do país de origem }\end{array}$ & $\begin{array}{l}\text { Mostre com clareza, a identificação } \\
\text { do país de origem }\end{array}$ \\
\hline d in an & Seja embalado ecologicamente & Seja embalado de forma que não \\
\hline & & \\
\hline
\end{tabular}




\section{3ª Etapa: Validação semântica}

Esta etapa visou identificar problemas de compreensibilidade dos itens existentes em um instrumento pela população à qual ele se destinava. Ressalta-se que Pasquali ${ }^{23}$ sugere que esse procedimento seja realizado em grupos com poucas pessoas (de três a quatro), visando garantir a fidedignidade das respostas.

Assim, a VP3 foi aplicada em uma amostra por conveniência de 26 estudantes universitários do sexto período de Enfermagem da Universidade Federal do Triângulo Mineiro (UFTM), sendo $96,2 \%$ mulheres, com média de idade 21,7 $\pm 2,7$ anos, Índice de Massa Corporal (IMC) 22,25 kg/ $\mathrm{m}^{2} \pm 4,62$, após assinatura do Termo de Consentimento Livre e Esclarecido (TCLE). Todos os participantes autorresponderam todos os itens. Todavia, grupos pequenos responderam também ao mesmo instrumento utilizado na etapa do comitê de especialistas ${ }^{22}$, que avaliava a compreensibilidade do texto, as pertinências e as sugestões. Sendo assim, cinco estudantes avaliaram os itens de um a seis (fator saúde); quatro, os itens de sete a 12 (fator humor); quatro, de 13 a 17 (fator conveniência); quatro, de 18 a 24 (fator apelo sensorial e fator conteúdo natural); cinco, de 25 a 30 (fator preço e fator controle de peso) e quatro participantes, de 31 a 36 (fator familiaridade e fator preocupação ética).

Os universitários não solicitaram auxílio dos pesquisadores para esclarecimento das questões, relataram que todos os itens eram de fácil entendimento e não fizeram nenhuma sugestão. Assim, a VP3 foi mantida, já que não houve modificações, sendo intitulada versão final em português do instrumento (VPF).

\section{4a Etapa: Pré-teste}

Esta etapa se destinou a obtenção de dados preliminares sobre as propriedades psicométricas e operacionais da escala, entre elas: verificar a consistência interna dos itens da versão adaptada, as medidas de distribuição e o tempo de duração da entrevista.

A VPF foi apresentada a uma amostra aleatória de 86 estudantes de ambos os sexos do segundo período dos cursos de Psicologia, Terapia Ocupacional e Engenharia. Os cursos foram selecionados por meio de sorteio entre os Institutos de Ciências Humanas, Ciências da Saúde e Ciências Exatas da UFTM. Para coleta dos dados de identificação e socioeconômicos, aplicou-se instrumento construído pelos próprios pesqui- sadores e foram inseridas questões referentes a medidas antropométricas (autorrelato de peso e altura) para o cálculo do IMC $\left(\mathrm{Kg} / \mathrm{m}^{2}\right)$. Dois estudantes não informaram o peso e foram excluídos do cálculo do IMC. A classificação do peso por meio do IMC utilizou pontos de corte propostos pela $\mathrm{WHO}^{24}$. Todos concordaram em participar de forma voluntária e assinaram o TCLE.

Para as análises estatísticas, utilizou-se o programa SPSS versão 20.0. Foram desenvolvidas análise descritiva (média, desvios-padrão) e a consistência interna dos itens de cada fator foi verificada segundo o coeficiente alfa de Cronbach, aceitando-se resultados superiores a $0,70^{25}$.

O projeto foi aprovado pelo Comitê de Ética em Pesquisa da UFTM, e o estudo está em conformidade com a resolução nº466/12 do Conselho Nacional de Saúde ${ }^{26}$.

\section{Resultados}

$\mathrm{Na}$ etapa referente à tradução, alguns termos não foram traduzidos literalmente, justamente pelo cuidado de contextualizar os vocábulos utilizados na versão original com seu significado, de acordo com a realidade cultural brasileira. Ressalta-se que nesta fase atentou-se para a escolha de palavras de fácil compreensão e de abrangência nacional. O item 17 , por exemplo, - is easily available in shops and supermarkets -, foi traduzido por "seja fácil de achar em mercearias e supermercados", por parecer mais adequado para a fluência da leitura. A expressão do item 27 - is good value for money - apresentou diferença entre as três tradutoras, duas delas optaram por "tenha um preço que eu considere justo" e a terceira "tenha bom custo-benefício". Assim, no consenso realizado pelas duas pesquisadoras nutricionistas, optou-se por "tenha o preço justo", com o intuito de garantir sua maior compreensão. A frase do item 34 - comes from countries I approve of politically - foi traduzida por "venha de países que eu aprove a forma como os alimentos são produzidos", e os itens 35 - has the country of origin clearly marked, e 36 - is packaged in an environmentally friendly way - foram traduzidos respectivamente por "mostre com clareza, a identificação do país de origem" e "seja embalado ecologicamente correto", por se adequar aos padrões brasileiros de comunicação.

$\mathrm{Na}$ etapa que contou com o comitê de especialistas, abriu-se uma discussão somente sobre a tradução dos itens 22 e 36 e com aprovação de todos os juízes eles foram modificados. As etapas 
de tradução e avaliação pelo comitê de especialistas podem ser observadas no Quadro 1.

No que tange ao pré-teste, participaram desta etapa 86 estudantes universitários, sendo 38 alunos da engenharia, 28 da psicologia e 20 da terapia ocupacional, com idades entre 18 a 71 anos, com média de $20 \pm 6,0$ anos e mediana de 19 anos.

Os estudantes necessitaram aproximadamente de 15 minutos para responder o instrumento. A amostra do pré-teste foi predominantemente composta por mulheres $(65,1 \%)$, eram solteiros $(96,5 \%)$, a maioria morava em república de estudantes $(45,3 \%)$, eram da raça branca $(79,1 \%)$ e relataram renda mensal familiar entre três e cinco salários mínimos (45,3\%), sendo que $68,6 \%$ não recebiam mesada fixa. Com relação à classificação de peso por meio do IMC, $11,9 \%$ dos estudantes apresentavam baixo peso $(\mathrm{n}=10) ; 61,9 \%$ tinham peso adequado $(\mathrm{n}=52)$; $17,9 \%$ estavam com sobrepeso $(n=15)$ e $8,3 \%$ apresentavam obesidade $(n=7)$.

No que concerne à pontuação do FCQ na etapa do pré-teste, maiores escores foram observados nos itens individuais "seja gostoso", seguido de "tenha um bom cheiro" e "tenha uma boa aparência" em que respectivamente $79,1 \%$, $54,7 \%$ e $50 \%$ dos estudantes consideraram muito importante. Todos estes três itens fazem parte do fator apelo sensorial. Ressalta-se que 50\% deles também responderam muito importante para o item "possa ser comprado em locais perto de onde moro ou trabalho". Por outro lado, os universitários relataram nada importante $(59,3 \% \mathrm{e}$ $52,3 \%)$ respectivamente às escolhas alimentares determinadas pelos itens "mostre com clareza a identificação do país de origem" e "venha de paí- ses que eu aprove a forma como os alimentos são produzidos", itens que compõe o fator preocupação ética.

No que se refere à consistência interna dos fatores do FCQ, verificou-se valores de alfa de Cronbach variando entre 0,75 (Preocupação Ética) a 0,89 (Conveniência e Controle de Peso), sendo o escore total do instrumento $\alpha=0,80$. Os valores de alfa de Cronbach de cada fator estão apresentados na Tabela 1 .

\section{Discussão}

O presente estudo descreve os procedimentos iniciais da adaptação do FCQ à língua portuguesa brasileira, uma vez que tais procedimentos que primam pela qualidade do instrumento são relevantes para garantir a veracidade e a qualidade das informações que serão investigadas em futuras pesquisas ${ }^{19,20,27}$, no contexto da nossa cultura.

Durante o processo de tradução, algumas expressões foram alteradas, porém o significado foi mantido, fato esse verificado no processo de retrotradução com o aceite do autor do instrumento original na sua totalidade.

$\mathrm{Na}$ avaliação pelo comitê de especialistas, a equivalência conceitual mostrou que poucas expressões necessitaram de ajustes e, na equivalência semântica, a versão traduzida mostrou que, dos 36 itens traduzidos, apenas dois $(5,5 \%)$ foram pouco modificados por todos os membros do comitê. A alteração no item 22 se deu porque a palavra "agrotóxicos" foi inserida na tradução sem fazer parte do instrumento original; e a modificação no item 36 devido ao pouco uso desta expressão (seja embalado ecologicamente correto)

Tabela 1. Consistência interna dos domínios do FCQ e do escore total para a amostra de universitários $(\mathrm{N}=86)$. Uberaba, MG, 2014.

\begin{tabular}{lcccc}
\hline \multicolumn{1}{c}{ Domínios do FCQ } & N $^{\mathbf{0}}$ de itens & Alfa de Cronbach & Média & Desvio-padrão \\
\hline Fator 1 - Saúde & 06 & 0,82 & 17,2 & $(4,0)$ \\
Fator 2 - Humor & 06 & 0,86 & 15,3 & $(4,9)$ \\
Fator 3 - Conveniência & 05 & 0,89 & 15,4 & $(4,2)$ \\
Fator 4 - Apelo sensorial & 04 & 0,86 & 13,4 & $(2,7)$ \\
Fator 5 - Conteúdo natural & 03 & 0,83 & 6,8 & $(2,5)$ \\
Fator 6 - Preço & 03 & 0,77 & 9,0 & $(2,3)$ \\
Fator 7 - Controle de peso & 03 & 0,89 & 7,2 & $(2,8)$ \\
Fator 8 - Familiaridade & 03 & 0,80 & 7,6 & $(2,4)$ \\
Fator 9 - Preocupação ética & 03 & 0,75 & 5,4 & $(2,1)$ \\
Escore total & 36 & 0,80 & 97,9 & $(12,4)$ \\
\hline
\end{tabular}


no cotidiano da população brasileira. A versão final do questionário manteve o formato e a sequência das questões igualmente a versão original do instrumento.

Na validação semântica, que identifica problemas de compreensibilidade dos itens do instrumento, os participantes não sugeriram nenhuma alteração. Assim, os autores julgam que o instrumento é relevante para a temática.

Com relação à aplicabilidade, todos os fatores e o escore total apresentaram boa consistência interna, com os valores de alfa de Cronbach superiores a $0,70^{25}$. Houve uma variação entre 0,75 a 0,89 para os fatores, corroborando o instrumento original inglês que obteve uma variação de 0,70 a $0,87^{9}$.

Apesar de não ser plausível comparar resultados do pré-teste desta pesquisa, pelo fato da amostra ser reduzida, com estudos utilizando o FCQ cujas amostras foram probabilísticas, resguardando as devidas diferenças metodológicas, a análise preliminar da presente pesquisa apontou que o menor escore se relacionou à preocupação ética, assim como no estudo britânico ${ }^{9}$ e na investigação europeia ${ }^{15}$. Entretanto, o fator mais importante diferiu, já que o fator saúde predominou entre os consumidores londrinos ${ }^{9}$, enquanto o apelo sensorial prevaleceu entre os demais europeus $^{15}$, assim como no atual estudo.

Diferenças são esperadas em virtude das diversidades interculturais ${ }^{19}$. Assim, estudar questões relacionadas à alimentação e nutrição em diferentes culturas pode ajudar a compreender os fatores influenciadores do comportamento alimentar ${ }^{28}$, sobretudo quando se leva em conta os fundamentos epistemológicos da alimentação, que se interagem entre a sociologia, antropologia, filosofia, psicologia, economia, política, além dos biológicos e nutricionais ${ }^{29}$.

O FCQ pode apresentar conotações distintas nas populações, mas mesmo assim, desde que adaptado ao idioma do lugar, pode ser um bom instrumento de medida para investigar sobre os motivos que norteiam as escolhas alimentares ${ }^{14}$.

Desta forma, com este estudo foi possível cumprir-se uma importante etapa para o processo de validação do FCQ, sendo que a versão traduzida está de acordo com o rigor das diretrizes de tradução e adaptação de outras medidas ${ }^{19-21}$, e a validade de face e de conteúdo foram realizadas. Faz-se ainda necessário aprimorar a validade de face em outra validação semântica composta de uma amostra heterogênea pelo menos quanto à escolaridade $\mathrm{e}^{23}$.
Ressalta-se que é igualmente importante avaliar sua reprodutibilidade, validade externa, equivalência operacional e de mensuração, a fim de comprovar suas propriedades psicométricas. Ambos os processos encontram-se em andamento numa amostra representativa da população brasileira, diversificada do ponto de vista etário, de gênero, socioeconômico, educacional entre outros, para que desta forma sua utilização seja indicada por outros pesquisadores em estudos epidemiológicos.

Por fim, apesar de o FCQ ser um instrumento que compreende vários importantes aspectos relacionados às escolhas alimentares, uma possível limitação é o fato dele não contemplar diretamente a influência da mídia (positiva ou negativamente) neste comportamento. Sabe-se que o marketing acerca dos alimentos, especialmente o televisivo, tem um poder persuasivo, atraente e duradouro na vida das pessoas ${ }^{30}$.

No entanto, ainda que exista esta pequena limitação, o presente instrumento abarca importantes fatores que permeiam as escolhas alimentares dos indivíduos. Além do mais, a metodologia utilizada para a tradução e verificação de sua adaptação cultural nesta primeira fase de validação do instrumento, mostrou-se adequada, pois cumpriu a finalidade de mensuração do objeto proposto, sendo de fácil entendimento à realidade brasileira.

\section{Colaboradores}

SFD Heitor foi a responsável pela elaboração do projeto, coleta e análise dos dados, redação e discussão do artigo. CCP Estima, FJ das Neves, ASA Nemer contribuíram na redação, na revisão crítica do artigo e aprovação da versão final. SS Castro e JES Ferreira participaram respectivamente como orientadora e coorientadora do projeto, análise dos dados, redação, revisão crítica do artigo e aprovação da versão final.

\section{Agradecimentos}

À Capes pela bolsa de doutorado de AS Aguiar. 


\section{Referências}

1. Jomori MM, Proença RPC, Calvo MCM. Determinantes de escolha alimentar. Rev. Nutr 2008; 21(1):63-73.

2. Brasil. Ministério da Saúde (MS). Coordenação-Geral da Política de Alimentação e Nutrição. Secretaria de Atenção à Saúde. Guia alimentar para a população brasileira: promovendo a alimentação saudável. Brasília: MS; 2006.

3. Brasil. Ministério da Saúde (MS). Coordenação-Geral da Política de Alimentação e Nutrição, Secretaria de Atenção à Saúde. Guia alimentar para a população brasileira. 2a ed. Brasília: MS; 2014.

4. Xavier HT, Izar MC, Faria Neto JR, Assad MH, Rocha VZ, Sposito AC, Fonseca FA, dos Santos JE, Santos RD, Bertolami MC, Faludi AA, Martinez TLR, Diament J, Guimarães A, Forti NA, Moriguchi E, Chagas ACP, Coelho OR, Ramires JAF. Sociedade Brasileira de Cardiologia. V Diretriz Brasileira de Dislipidemias e Prevenção da Aterosclerose. Arq Bras Cardiol 2013; 101(4 Supl. 1):1-22.

5. World Health Organization (WHO). Global health risks: mortality and burden of disease attributable to selected major risks. Geneva: WHO; 2009.

6. Canella DS, Levy RB, Martins APB, Claro RM, Moubarac JC, Baraldi LG, Cannon G, Monteiro CA. Ultra-Processed Food Products and Obesity in Brazilian Households (2008-2009). Plos One 2014; 9(3):e92752.

7. Souza AM, Pereira RA, Yokoo EM, Levy RB, Sichieri R. Alimentos mais consumidos no Brasil: Inquérito $\mathrm{Na}$ cional de Alimentação 2008-2009. Rev Saude Publica 2013; 47(Supl. 1):190S-199S.

8. Instituto Brasileiro de Geografia e Estatística (IBGE). Pesquisa de Orçamentos Familiares 2008-2009: análise do consumo alimentar pessoal no Brasil. Rio de Janeiro: IBGE; 2011.

9. Steptoe A, Pollard TM, Wardle J. Development of a measure of the motives underlying the selection of food: the Food Choice Questionnaire. Appetite 1995; 25(3):267-284.

10. Ferreira VA, Magalhães R. Práticas alimentares cotidianas de mulheres obesas moradoras da Favela da Rocinha (Rio de Janeiro, RJ, Brasil). Cien Saude Colet 2011; 16(6):2983-2991.

11. Poulain JP, Proença RPC. Reflexões metodológicas para o estudo das práticas alimentares. Rev. Nutr 2003; 16(4):365-386.

12. Minayo MCS, organizador. Pesquisa social: teoria, método e criatividade. 29a ed. Rio de Janeiro: Vozes; 2010.

13. Pieniak Z, Verbeke W, Vanhonacker F, Guerrero L, Hersleth M. Association between traditional food consumption and motives for food choice in six European countries. Appetite 2009; 53(1):101-108.

14. Jáuregui-Lobera I, Ríos PB. What motivates the consumer's food choice? Nutr. Hosp 2011; 26(6):1313-1321.

15. Januszewska R, Pieniak Z, Verbeke W. Food choice questionnaire revisited in four countries. Does it still measure the same? Appetite 2011; 57(1):94-98.

16. Pieniak Z, Pérez-Cueto F, Verbeke W. Association of overweight and obesity with interest in healthy eating, subjective health and perceived risk of chronic diseases in three European countries. Appetite 2009; 53(3):399406.
17. Ares G, Gámbaro A. Influence of gender, age and motives underlying food choice on perceived healthiness and willingness to try functional foods. Appetite 2007; 49(1):148-158.

18. Cohen L, Manion L, Morrison K. Research Methods in Education. $5^{\text {th }}$ ed. London: Routledge Falmer; 2000.

19. Guillemin F, Bombardier C, Beaton D. Crosscultural adaptation of health-related quality of life measures: literature review and proposed guidelines. J Clin Epidemiol 1993; 46(12):1417-1432.

20. Beaton DE, Bombardier C, Guillemin F, Ferraz MB. Guidelines for the process of cross-cultural adaptation of self-report measures. Spine 2000; 25(24):3186-3191.

21. Beaton DE, Bombardier C, Guillemin F, Ferraz MB. Recommendations for the cross-cultural adaptation of health status measures. Washington: American Academy of Orthopaedic Surgeons, Institute for Work \& Health; 2002.

22. Disabkids Group. Translation and validation procedure: guidelines and documentation form. Leiden: The Disabkids Group; 2004.

23. Pasquali L. Princípios da elaboração de escalas psicológicas. Rev. Psiq Cli 1998; 25(5):206-213.

24. World Health Organization (WHO). Obesity: preventing and managing the global epidemic. Geneva: WHO; 1998. (Report of a WHO consultation on obesity).

25. Terwee CB, Bot SDM, Boer MR, van der Windt DAWM, Knol DL, Dekker J, Bouter LM, de Vet HCW. Quality criteria were proposed for measurement properties of health status questionnaires. J Clin Epidemiol 2007; 60(1):34-42.

26. Brasil. Ministério da Saúde (MS). Conselho Nacional de Saúde. Resolução no 466, de 12 de dezembro de 2012. Diário Oficial da União 2013; 13 jun.

27. Reichenheim ME, Moraes CL. Operacionalização de adaptação transcultural de instrumentos de aferição usados em epidemiologia. Rev Saude Publica 2007; 41(4):665-673.

28. Estima CCP, Bruening M, Hannan PJ, Alvarenga MS, Leal GVS, Philippi ST, Neumark-Sztainer D. Cross-Cultural Comparison of Eating Behaviors and Home Food Environmental Factors in Adolescents From São Paulo (Brazil) and Saint Paul-Minneapolis (US). J Nutr Educ Behav 2014; 46(5):370-375.

29. Prado SD, Bosi MLM, Carvalho MCVS, Gugelmin SA, Silva JK, Delmaschio KL, Martins MLR. A pesquisa sobre Alimentação no Brasil: sustentando a autonomia do campo Alimentação e Nutrição. Cien Saude Colet 2011; 16(1):107-119.

30. Santos CC, Stuchi RAG, Arreguy-Sena C, Pinto NAVD. A influência da televisão nos hábitos, costumes e comportamento alimentar. Cogitare Enferm 2012; 17(1):6571.

Artigo apresentado em 09/10/2014

Aprovado em 29/01/2015

Versão final apresentada em 31/01/2015 